\title{
ALLELIC COMPLEMENTATION RECONSIDERED
}

by

\author{
JOHN R. S. FINCHAM
}

\author{
Department of Genetics, University of Edinburgh, \\ West Mains Road, Edingburgh EH9 3JN, Scotland \\ Emil Chr. Hansen lecture delivered at the \\ Carlsberg Laboratory on September 22nd, 1977.
}

\section{INTRODUCTION}

Althoug it has, during the whole of the present century, been obvious that heredity has to be interpreted in terms of discrete genetic units or genes, there has always been some ambiguity, at first concealed but latterly increasingly obvious, surrounding the question of how the gene could be or ought to be defined. During the heyday of classical cytogenetics it was usually assumed that the gene was both a unit of function, as defined by what we would now call failure of defective alleles to complement one another and, at the same time, an indivisible unit of structure as defined by failure of its alleles to yield recombinants from interallele crosses. Genetics certainly got a long way without the non-equivalence of the functional and recombination units becoming obvious, but strain began to appear with the discovery of "pseudoalleles" independently by E. B. LEWIS and M. M. GREEN in Drosophila, and the apparently smooth facade of the classical allpurpose gene collapsed completely with the fine-structure analyses of PONTECORvo, ROPER and PRITCHARD on the fungus Aspergillus and of BENZER on bacteriophage T4*. Briefly, what these investigators found was that independently isolated mutants, classified as carrying defective alleles of the same gene by the criterion of non-complementation, could in general yield wild type recombinants in crosses with each other, though at low frequencies. Thus the unit of function comprised many units of recombination. BENZER (1957) set the seal on this fundamental discovery by proposing a new reformed terminology in which the functional unit was called the cistron and the recombinational unit a recon. Over the following decade it emerged that the cistron was, most usually at any rate, the DNA sequence coding for a single polypeptide chain - some hundreds or thousands of base pairs - while the recon was nothing larger than a single base pair (BENZER's third unit, the muton, was never distinguished operationally from the recon and the two units eventually turned out to be the same thing).

Hardly, however, had BENZER's elegantly simple neo-classical structure been erected when new cracks began to appear. Most geneticists would have been happy to accept a

*For review see Fincham (1966) 
redefinition of the gene (an obstinately tenacious word) as the cistron, placing reliance on the complementation criterion and accepting the occurrence of recombination within genes. Unfortunately for their peace of mind, extensive analyses of series of mutants in Neurospora showed that occasional complementation between pairs of mutants which, on the basis of the fact that they were defective in the same enzyme, were expected to be mutant alleles of the same gene, did nevertheless complement one another (FINCHAM \& PATEMAN, 1957; WoODWARd et al., 1958; CATCHESIDE \& OVERTON, 1959). In cases of this kind the series of mutants could not be divided into neat sub-groups which would qualify as cistrons in their own right because many or most mutants of the series were usually found to fail to complement with any of the others. What emerged was, in fact, a "messy" cistron in which certain mutants showed complementation, in a pattern which could usually be represented by a more or less complex complementation map, but many or most did not; these latter provided a series of overlaps on the complementation map which, as it were, tied the whole series of mutants together. Such a series, defined by the combined span of the overlaps, was soon accepted as the rightful inheritor of the title "gene" and the members of such a series were considered to be allelic. In many cases such a series was shown to correspond to a single enzyme. In order to salvage the cistron concept and make it correspond to what nearly everybody wanted to recognize as the functional gene, it was necessary to modify the definition so that only complete or perfect complementation should be taken as evidence for different cistrons. Imperfect complementation, to give a more or less defective or abnormal product, was to be recognized as compatible with the mutations concerned being in the same cistron in the revised definition. This proposal was partly based on experimental results but partly also on faith; it did indeed turn out in my own system the am mutants of Neurospora controlling glutamate dehydrogenase - that complementation between mutant alleles gave recognizably defective even though functional enzyme (FINCHAM, 1959) but it had to be admitted that the imperfection of the complementation product was not by any means always easy to demonstrate even though theory demanded it. We nevertheless held to the theory because, short of postulating quite unknown and seemingly improbable molecular events such as recombination of RNA molecules, there was just no alternative available model of what might be happening. It seemed almost inescapable that the gene (represented by a complementation map tied together by overlaps) coded for a single polypeptide chain, that the enzyme product of the gene was an oligomer containing, in the wild type 2, 4, 6 or some other number of identical chains, and that, in the complementation situation, two different kinds of defective chains were being included together in the same oligomer and in some way (supposedly by mutual correction of conformation) were making good each other's shortcomings. On this model, which was probably first proposed by CATCheside (CATCHEside \& Overton, 1959), it was clear that the complementation product must be structurally abnormal, containing two different kinds of bad chain rather than one kind of good one, however well it managed to conceal the fact.

It will be from this rather sketchy historical outline that the theory of allelic complementation, as it was formulated in the early 1960's, contained assumptions both about the molecular nature of gene action and about the nature of the complementary interaction of polypeptide chains in mixed oligomers. It is my purpose in this lecture to consider where we stand today in relation to each of these aspects of complementation theory.

\section{COMPLEMENTATION AND THE DEFI- NITION OF THE GENE}

Almost from the first, the cistron was supposed to code for a single polypeptide chain; it seemed self-evident that this would be the smallest unit of gene translation which would be at all likely to be functional. When the strict cistron was superseded by the reformed, or broad, cistron, the latter inherited the same 
presumed function. Indeed, this view of the matter is still not out of date, and we are still, at least in my opinion, most likely to be correct if we equate the broad cistron whit the unit (call it the gene) coding for one polypeptide chain. There are, however, pitfalls in the application of this concept.

The first source of confusion and possible error is the existence, which has come to be increasingly appreciated during the last few years, of multiheaded enzymes. These functionally complex proteins have different, separately assayable, enzyme activities associated with different regions of their surfaces - often, it seems with different, separately folded "domains" of tertiary structure. In such cases it has often been controversial whether the multiheaded enzyme consists of an aggregate of different polypeptide chains, each associated with a single activity, or whether the different activities are functions of a single kind of polypeptide chain folded to form a number of quasi-independent domains. It begins to look as if the argument will be resolved in nearly all cases in favour of the single-chain alternative. Examples in which genetic analysis has been followed by analysis of the structure of the protein gene product are the complex enzymes responsible for fatty acid synthesis in yeast (KOBLING et al., 1975), three steps in histidine synthesis in yeast (BIGELIS et al., 1977), the early steps in pyrimidine synthesis in Neurospora (MAKOFF, 1977), and the synthesis of aromatic amino acids in Neurospora (GAERTNER \& COLE, 1977). In each of these cases it is easy to eliminate the activity of one part of the complex while leaving that of the others intact and, if attention is concentrated on missense mutants (single amino acid changes) one sees a number of non-overlapping complementation groups each corresponding to a single enzyme activity. Complementation between members of different groups is usually complete. It would be easy to conclude, and it was so concluded until recently, that in each of these systems there is a cluster of genes coding for a number of polypeptide chains responsible for sequentially acting (or at least related) enzyme activities. What cast doubt on this interpretation was the existence of a number of mu- tants with multiple enzyme deficiencies. These, now known to be chain-termination (nnonsense") mutants, overlapped some or alle of the single-enzyme-activity groups in the complementation map in a polarized pattern (CASE \& Giles, 1968; Shaffer et al., 1969). At first we tended to interpret these "polar" or pleiotropic mutants in terms of polar effect within an operon; the principal difficulty with this interpretation was that there was no obvious mechanism, and no precedent in the relatively well understood bacterial operons, for the absolute polar effect exercised by chain terminations on the all distal activities. The need for postulating a new kind of very stringent polar effect of a nonsense mutant in one gene on the expression of all of a set of more distal genes has, however, largely disappeared; in each of the cases I have mentioned it now seems very likely, if not quite certain, that we are dealing with a single polypeptide chain which folds itself to make a multiheaded enzyme with a number of functionally rather independent domains. Absolute translational polarity within a single chain is, of course, entirely expected. Given the rather surprising complexity of function of some of these single chains, the finestructure mapping and complementation data are entirely understandable. So far as eukaryotes are concerned, single genes coding for polypeptides of complex function are at present very much in favour, and there seems to be no clear evidence for the existence of operons - that is, sequences with several units of translation within one unit of transcription. If eukaryote operons exist, in the sense of polygenic units of transcription, their component genes are apparently translated independently without polar effects.

Where, then, does this leave the problem of defining genes on the basis of complementation data? Form the practical point of view the situation is far from satisfactory, in that it is only chain terminating mutants, and the overlaps provided by them in the complementation map, which distinguish a single gene coding for a multiheaded enzyme from a gene cluster. If one is unlucky enough not to recover chain-termination mutants in the translationproximal regions of the gene one may easily be 
misled into the mistaken diagonosis. The only way to make this kind of mistaken interpretation improbable is to collect a sufficiently large sample of mutants. Ideally, one should confirm the chain-terminating nature of the crucial pleiotropic (non-complementing) mutants by showing (a) that they are not deletions, the criteria here being revertibility and finestructure recombinational mapping, and (b) that they are suppressed by known suppressors of chain termination. In the examples from yeast and Neurospora mentioned above these additional checks have been carried out, but the whole analysis requires a set of time-consuming tests which are far more clumsy and troublesome than BENZER's original elegant and rapid procedures for functionally simple cistrons.

In the foregoing discussion I have been assuming that the gene we are after is the unit of translation - what one might call the protein gene. What of the relation between this unit and the unit of transcription - the RNA gene? It is perfectly possible that complementation data may give us the latter with the former included within it. If there is a promoter region concerned with initiation of transcription outside the DNA sequence which codes for amino acid sequence, one will expect to find a class of noncomplementing mutants, which will be pleiotropic in the case of a gene of complex function, mapping beyond the protein gene at its "N-terminal" end. CHovNick and his colleagues (CHOVNICK et al., 1976) have established an excellent case for the existence of such a region for the rosy gene of Drosophila (coding for xanthine dehydrogenase). In fact the gene as defined as the genetic segment occupied by mutually non-complementing mutants may in principle consist of three regions: (a) necessary for transcription initiation but itself not transcribed (so far a conjectural region but certainly a possible one); (b) transcribed but not translated (like promoter mutational sites in the lac operon of E.coli); and (c) the protein structural gene proper. Perhaps we need some innovations in terminology to take account of these likely subdivisions of the gene; the point which I wish to make here, however, is that complementation tests are not enough to discriminate between them; only laborious study of the macromolecular gene products and their alterations in mutants can finally confirm the type of genetic unit we are dealing with.

\section{THE MECHANISM(S) OF ALLELIC COMPLEMENTATION}

In cases where a gene specifies a complex enzyme with two or several activities, and we are dealing with mutant alleles which have each lost just one activity while retaining the other(s), there is obviously no problem in explaining allelic complementation. This kind of situation is commoner than we once thought. The interesting questions, however, are posed by those many examples in which the complementing alleles each produce no detectable enzyme activity of any kind.

What is the mechanism, or mechanisms, whereby mutant alleles which, by themselves, produce entirely inactive polypeptide chains, manage to complement one another, at least partially, in heterozygous diploids or heterokaryons? Our view of this question has changed very substantially since the 1960 's. The general mechanism put forward then, in most explicit detail by CRICK and Orgel (1964), can be summarised as conformational correction, operating between complete mutant monomers in hybrid oligomers. The idea was that a conformationally defective region of one mutant monomer could be corrected by a rather direct contact with the homologous correctly folded region of a mutant monomer with a different defect. Though the general idea of conformational correction is, in my view, still valid in many cases, it can, and has been, criticised from several points of view. In an interesting paper published a few years ago SCHWARTZ (1975) has argued against correction on the basis of his own results on heteroallelic interaction in the formation of alcohol dehydrogenase in maize. His results indicated not so much correction as the prevention in hetero-oligomers (tetramers in maize $\mathrm{ADH}$ ) of the denaturation which tended to occur when the defective monomers were aggregated only with their own kind. Accepting SCHWARTZ's interpretation here, it still does not seem to me that a basically 
different idea is involved. One partner in the complementary interaction is still supposed to stabilize the other in the correct conformation, and this is the essence of the original concept; the stabilization may indeed be pre-emptive (preventing a deformation from occurring) rather than remedial (correcting it after it has happened), but these two apparent alternatives are surely only two different ways of looking at the same conformational transition which must, in itself, be reversible.

Quaternary restraints operating between monomers of a protein oligomer have now been directly demonstrated in a number of instances, the general tendency being the imposition of conformational uniformity among the monomers. Normalisation of the conformation of a defective mutant monomer by hybridisation with its wild type (or conformationally more normal) counterparts has been shown in a number of cases including our own system, Neurospora glutamate dehydrogenase. There is no doubt that conformational correction (or stabilization) is an important mechanism of allelic complementation. The questions which need to be looked at again are how it occurs and whether it accounts for as great a part of the total phenomenon of allelic complementation as we originally thought.

To deal with the first question first, it now looks as if the model of CRICK and ORGEL (1964), the essence of which was local interaction ("good corrects bad") between homologous sections of monomers where they came into juxtaposition near a dyad axis, was too special, and, in a sense, too logical. The reason for the emphasis on interactions near a dyad axis of symmetry was the argument that, if the defect in one complementing monomer was away from the axis the faulty region would be juxtaposed with a non-homologous and normal region even in a homo-dimer, in which case why did not the mutant complement itself? The difficulty nowadays with the CRICK-ORGEL model is that it leads to expectations about the architecture of dimeric and oligomeric proteins which are not borne out by the numerous threedimensional protein structures which are now known. As RATNER and RoDIN (1977) have recently pointed out in a thoughtful review of the whole subject of allelic complementation, very few of the contacts at intermonomer interfaces involve homologous residues. I believe that, rather than thinking in terms of a series of independently deformable and correctable regions along a dyad axis, it makes more sense to base an interpretation on alternative conformational states of the monomer as a whole, the equilibrium between which can be shifted by many different amino acid replacements in various parts of the three-dimensional structure. Restraints operating between monomers in a hybrid oligomer may often prevent a mutant monomer from assuming the abnormal conformation which it would adopt in aggregation with its own kind - this may be a matter of a conformationally "correct" monomer (perhaps inactive for some non-conformational reason) correcting a conformationally faulty one, or of two conformationally faulty monomers tending to deform the oligomer in opposite directions and hence to some extent cancelling each other's defects. The idea of "two blacks making a white - rejected by CRICK and ORGEL because it did not predict the simple complementation maps then thought to be the rule - may well have validity as one of several kinds of conformational interaction which combine to generate the very complex complementation maps which are now commonly found. As more extensive series of mutants have been analysed, in fact, the necessity for explaining why complementation maps should be relatively simple and generally linear has become much less pressing, since it has become evident that the premise is false; simplicity usually only persists so long as the number of mutants to be accommodated is relatively small. Geometrical complexity seems to be the rule for complementation maps, and one may well wonder whether the degree of order that they do seem to display is much more than one could expect to occur by chance. This is not to say that they are not a useful way of summarizing data, but it seems to me that they will only be related in detail to actual protein conformation after the conformation is already known from $\mathrm{X}$-ray crystallography; the relation between con- 


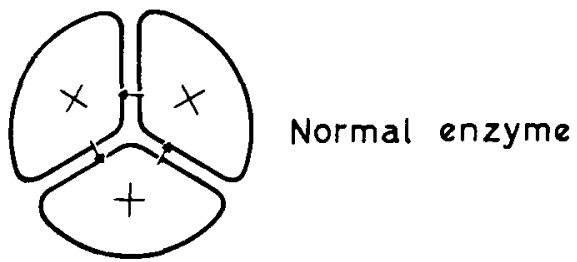

Mutant products

Examples of

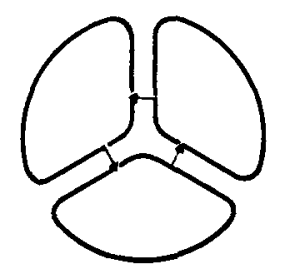

1

No coenzyme binding

complementation products

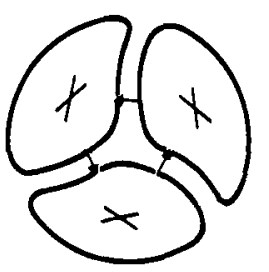

\section{Abnormal \\ 3 tertiary}

structure

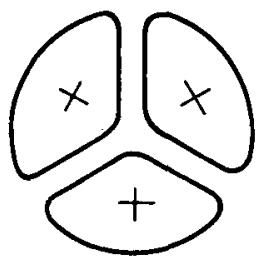

Unstable
14 quaternary
structure
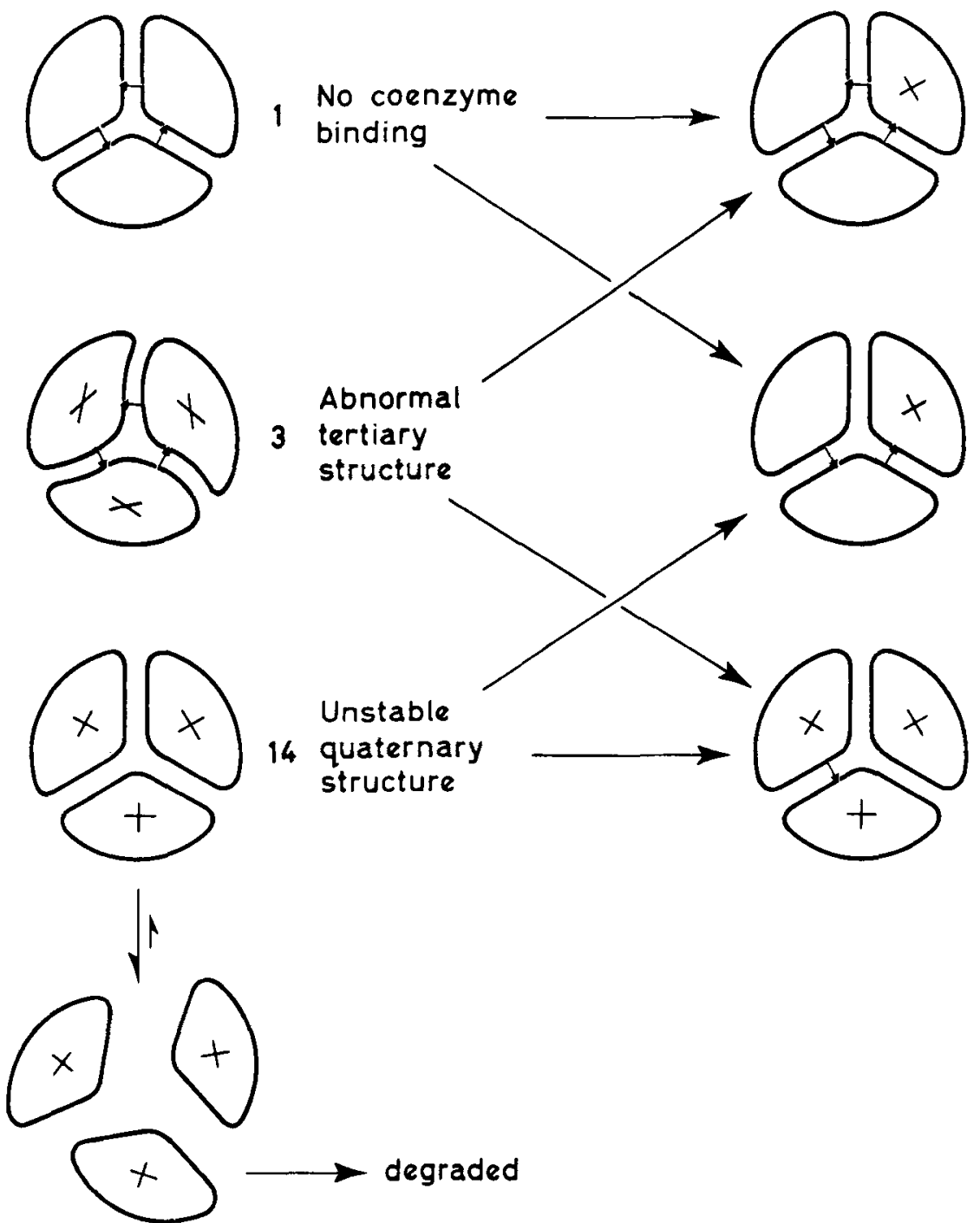

Figure 1. Models for complementation between mutants 1,3 and 14 , defective respectively in coenzyme-binding site, tertiary conformation and quaternary aggregation. The enzyme is represented as a trimer, but is in fact a hexamer - perhaps two apposed trimers related by a dyad axis. The coenzyme binding site (missing in 1) is represented by a cross and an inter-monomer stabilizing contact (missing in 14) by an arrow. 
formation and map will probably turn out to be special for each protein.

A recent approach to making some sense out of complementation maps, has been developed by RATNER and RODIN. These authors have used computer methods to sort out each of several extensive series of allelic mutants into main groups so as to maximise intergroup and minimise intra-group complementation while at the same time limiting the number of groups. They believe that the main groups so defined can be related to distinct functions of the protein gene product - either different enzymic functions, when the protein possesses more than one, or distinct structural features necessary for the same enzymic activity. Occasional failures to complement between the main groups or occasional occurrences of complementation within them, can have special explanations and are not given much weight in relation to the main pattern.

The system on which I and my colleagues have worked - that of Neurospora glutamate dehydrogenase - can I believe, be interpreted rather well, in terms of three general functions of the protein (B RETT et al., 1976; FinCHAM \&

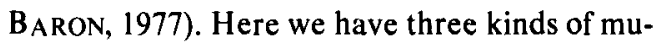
tants which complement one another in all three pairwise combinations. These three, which are typified by mutants, 1,3 and 14 , are known to represent respectively defective coenzyme binding, grossly disturbed allosteric equilibrium and failure of stable quaternary association. It is quite understandable that these mutants, in so far as their defects are confined to the features just mentioned, should complement one another in hetero-oligomers; Figure 1 explains how we picture these interactions as occurring. It is also not surprizing that certain mutants "overlap" two of the three main groups; it is easy to see in principle that an amino acid replacement could affect two of the three main properties while leaving the third more or less normal. Our interpretation here is only made possible by the results of detailed studies of the properties of the mutant proteins produced by various mutants. The complementation map begins to become interpretable and to acquire a certain predictive value after a good deal is known of the properties of the wild

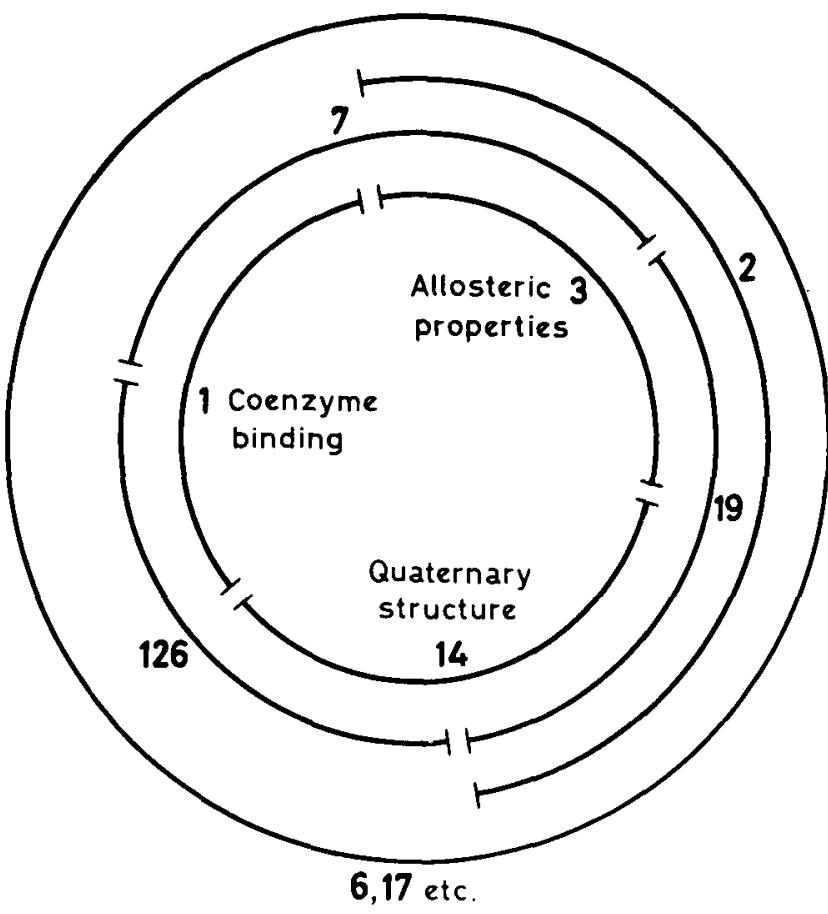

Figure 2. Complementation map of the am gene of Neurospora crassa. Mutant 126, hitherto not reported, was from the recent collection of Dr. J. A. KINSEY, and kindly made available by him for complementation testing. It appears to resemble 14 in producing no detectable mutant protein under normal conditions except when complemented by another mutant, but it differs from 14 in not being osmotically reparable (cf. FINCHAM \& BARON, 1977).

type and several mutant enzyme varieties. The fact that the complementation map (Figure 2) has now become circular (after a long period of two-ended linearity) should not be given any great significance; there is probably no finality about the circle and, indeed, the recent discovery of one mutant which shows weak complementation with mutants 1 and 19, but with no others, would require the folding of the circle to form a complex three-dimensional figure if we were to include it in the complementation map.

My argument so far can be summarized by saying that our first ideas about mechanisms of complementation between monomers in a mixed oligomer were to narrow and formalistic, taking inadequate account of the variety and 
complexity of the functions and interactions within protein oligomers. It is also true that we greatly underestimated the possibilities for complementation involving fragments of polypeptide chains. Our prejudice against fragment complementation, which was really based on little more than ill-founded intuition, was undermined from two directions. Firstly, the polarized patterns seen in many complementation maps, for example those for ad-3B (DE SERREs et al., 1967) and pyr-3 (RADFORD, 1969) in Neurospora, were interpreted by the authors concerned as due to the participation of incomplete chains coded for by nonsense mutants. This intepretation was substantiated in the cases of the Neurospora aro and yeast his 4 "gene clusters" (in reality, as I have already argued, single genes coding for polypeptides of complex function). In these cases it was shown that the complementing mutants which gave polarized overlaps really were chainterminators by the criterion of suppressibility. From protein chemistry also, evidence has accumulated in recent years that fragment complementation, in which a complete and normal primary sequence is pieced together from fragments of the polypeptide chain, is not only possible but rather common, and often very efficient. The classical case, which was already published at the time we were putting all the emphasis on complete chain interactions, was that of RICHARDS (1958) on pancreatic ribonuclease and its subtilisin fragment. The most detailed work in this area was carried out more recently by ANFINSEN and his group with the nuclease of Staphylococcus aureus (ONTJES \& ANFINSEN, 1969). Other excellent examples, which can be related to $\mathrm{E}$. coli genes which have been mapped in detail by genetic methods, are provided by $\beta$-galactosidase (GOLDBERG, 1969) and tryptophan synthetase (JACKSON \& Y ANOFSKY, 1969). In each of these cases a complete and fully functional folded structure can be formed in vitro (and, where tested, in vivo also) from complementary fragments of the polypeptide chain. The fragments normally have to be overlapping, and in this case the reconstituted functional product has an extra staila of redundant sequence which can it seems, be accommodated on the outside of the structure without interfering unduly with stability or enzymic function. In the case of Staphylococcal nuclease even non-overlapping fragments may work; fully active enzyme can be reconstituted by interaction between fragments containing residues $6-48$ on the one hand and either $49-149$ (the C-terminus), or 50-149 on the other. It seems that many globular proteins consist of separately folded "domains" held together not so much by the continuity of primary structure as by quaternary, probably mainly hydrophobic, bonds of much the same kind as are involved in holding together the separate chains of proteins which are normally ologomeric. The stability of the quaternary structure may be relatively little affected by nicking of the primary sequence in between domains. I referred above to the difficulties which this kind of secondary fragmentation of complex enzymes, which are primarily homooligomers of single polypeptide chains, has posed in analyses of "complex locin.

\section{DOES ALLELIC COMPLEMENTATION HAVE ANY SIGNIFICANCE IN NATURE?}

I have explained how allelic complementation posed a challenging puzzle to geneticists interested in defining genetic units and understanding gene action, and also how it can afford som intriguing insights into the structures of complex protein molecules and the way they respond to structural deformations of various kinds. All this is interesting, but does it have any bearing on the world outside the laboratory? I proposed some years ago (FINCHAM, 1972) that since heterooligomeric enzymes, determined by heterozygotes were undoubtedly often different in properties from the corresponding homooligomers, they might sometimes be better, even in cases where the alleles concerned were each functional nwild typesi. On the other hand, as Zouros (1976) has recently pointed out, there is no indication in available data that multimeric enzymes are more polymorphic than monomeric ones quite the contrary in fact. This does not, of course, entirely dispose of the possibilility that hetero-oligomeric molecules may occasionally be superior to their homooligomeric counter- 
parts even though the more usual case may be that they are inferior. We have found (FInchaM, 1972; J. C. WootTon et al., unpubl.), using mutants of Neurospora am series, that hetero-oligomers formed from GDH varieties with very different allosteric constants show a range of intermediate properties. In general the combination, in heterokaryon or heterozygote, of two such different types of monomer will yield enzyme which, as a whole, is less tightly controlled (i.e. exhibits its allosteric transition over a broader range of metabolite concentration) than either of the two homokaryons or homozygotes. This may be either because allosteric transitions within mixed oligomers are not fully concerted, or because of the formation of a range of heterooligomers of different compositions, or for both reasons. No doubt this relaxed control will in general be less than optimally adapted to any particular habitat and climate, but it is conceivable that it could be an advantage at a time of changing conditions, when old systems of control might be breaking down and new ones not yet fixed. The whole question of whether, and if so how often, hetero-oligomeric enzymes are positively selected for has still to be settled by experimental and field observation. Some existing proteins, of which haemoglobin and animal lactate dehydrogenase are the prime examples, are dimers of obviously homologous polypeptide chains now coded for by different genes. The hypothesis that these represent the stabilisation by gene duplication of what in the first instance was the favourable interaction of two alleles originally of the same gene, remains a rather obvious and attractive hypothesis. Such episodes in protein evolution might, however, be too rare to make it very likely that one would catch an example in a limited sample of the ubiquitous - and probably largely inconsequential - enzyme polymorphisms seen in nature.

\section{REFERENCES}

1. BENZER, S.: The elementary units of heredity. In: The Chemical Basis of Heredity, W. D. McElroy \& B. Glass eds., Johns Hopkins Press, pp. 70-93 (1957)
2. Bigelis, R., J. KeEsey \& G. R. FinK: The his 4 fungal gene cluster is not polycistronic. (1977)

3. Brett, M., G. K. Chambers, A. A. Holder, J. R. S. FINCHAM \& J. C. WOOTTON: Mutational amino replacements in Neurospora crassa NADPspecific glutamate dehydrogenase. J. Mol. Biol. 106, 1-22(1976)

4. CASE, M. E. \& N. H. GILES: Evidence for nonsense mutation in the arom gene cluster of Neurospora crassa. Genetics 60, 49-58 (1968)

5. Catcheside, D. G. \& A. Onerton: Complementation between alleles in heterokaryons. Cold Spring Harb. Symp. Quant. Biol. 23, 137-140 (1959)

6. Chovnick, A., W. Gelbart, M. McCarron, B. Osmond, E. P. M. CANDido \& D. L. BaIllie: Organization of the rosy locus in Drosophila melanogaster: Evidence for a control element adjacent to the xanthine dehydrogenase structural element. Genetics 84, 233-255 (1976)

7. Crick, F. H. C. \& L. E. Orgel: The theory of inter-allelic complementation. J. Mol. Biol. 8, 161165 (1964)

8. De Serres, F. J., H. E. Brockman, W. E. BarNETT \& H. G. KøLMARK: Allelic complementation among nitrous acid-induced mutants of Neurospora crassa. Mutation Res. 4, 415-424 (1967)

9. FinCHAM, J. R. S.: On the nature of the glutamic dehydrogenase formed by inter-allelic complementation at the am locus of Neurospora crassa. J. Gen. Microbiol. 31, 600-611 (1959)

10. Fincham, J. R. S.: Genetic Complementation. pp. 143, W. A. Benjamin Inc. New York (1966)

11. Fincham, J. R. S.: Heterozygous advantage as a likely general basis for enzyme polymorphisms. Heredity 28, 387-391 (1972)

12. Fincham, J. R. S. \& A. J. Baron: The molecular basis of an osmotically reparable mutant of Neurospora crassa producing unstable glutamate dehydrogenase. J. Mol. Biol. 110, 627-642 (1977)

13. Fincham, J. R. S. \& J. A. Pateman: Formation of an enzyme through complementary action of mutant "alleles in separate nuclei in a heterocaryon. Nature, Lond. 179, 141-142 (1957)

14. Gaertner, F. H. \& K. W. Cole: A cluster-gene: Evidence for one gene, one polypeptide chain, five enzymes. Biochem. Biophys. Res. Comm. 75, 259-264 (1977)

15. GoldberG, M.: Tertiary structure of Escherichia coli $\beta$-galactosidase. J. Mol. Biol. 46, 441-446 (1969)

16. JACKSON, D. A. \& C. YANOFSKY: Restoration of enzymatic activity by complementation in vitro between mutant a-suburits of tryptophan syn- 
thetase and between mutant subunits and fragments of the a-subunit. J. Biol. Chem. 244, 4539$4546(1969)$

17. Kobling, A., D. Schiffmann, H. D. Sickinger \& E. SChweitzer: Malonyl and palmityl transferase-less mutants of the yeast fatty-acidsynthetase complex. Eur. J. Biochem. 56, 359-367 (1975)

18. MAKofF, A. J.: Characterization of the aspartate carbamyl-transferase fragment generated by protease action on the pyrimidine-3 gene product og Neurospora crassa. Biochim. Biophys. Acta., in press.

19. ONTJES, D. A. \& C. B. ANFInSEN: Synthetic studies of structure function relationships in staphylococcal nuclease. J. Biol. Chem. 244, 6316-6322 (1969)

20. Radford, A.: Polarized complementation at the pyrimidine-3 locus of Neurospora. Molec. Gen. Genet. 104, 288-294 (1969)
21. Ratner, V. A. \& S. N. Rodin: Theoretical aspects of genetic complementation. Prog. Theoret. Biol. 4, 1-63 (1977)

22. RichardS, F. M.: On the enzymic activity of subtilisin-modified ribonuclease. Proc. Nat. Acad. Sci., USA 44, 162-166 (1958)

23. Schwartz, D.: The molecular basis for allelic complementation of alcohol dehydrogenase mutants of maize. Genetics 79, 207-212 (1975)

24. Shaffer, B., J. Rytka \& G. R. Fink: Nonsense mutations affecting the his 4 enzyme complex of yeast. Proc. Nat. Acad. Sci., USA 63, 1198-1205 (1969)

25. Woodward, D. O., C. W. H. Partridge \& N. H. GILES: Complementation at the ad-4 locus in Neurospora crassa. Proc. Nat. Acad. Sci., USA 44, 1237-1244. (1958)

26. Zouros, E.: Hybrid molecules and the superiority of the heterozygote. Nature 262, 227-229(1976) 